OPEN ACCESS

Edited by:

Ivana Rosenzweig, King's College London,

United Kingdom

Reviewed by:

Mikhail G. Poluektov,

I.M. Sechenov First Moscow State

Medical University, Russia

Michelangelo Maestri,

University of Pisa, Italy

*Correspondence:

Xiangyang Zhang

zhangxy@psych.ac.cn

Meihong Xiu

xiumeihong97@163.com

†These authors have contributed equally to this work

Specialty section: This article was submitted to

Sleep Disorders,

a section of the journal

Frontiers in Psychiatry

Received: 03 September 2020 Accepted: 13 November 2020 Published: 07 December 2020

Citation:

Liu D, Liu S, Zhu L, Li D, Huang D, Deng $H$, Guo $H$, Huang D, Liao Y,

Mao Z, Miao Q, Liu W, Xiu M and

Zhang $X$ (2020) Prevalence and Related Factors of Insomnia Among Chinese Medical Staff in the Middle and Late Stage of COVID-19.

Front. Psychiatry 11:602315.

doi: 10.3389/fpsyt.2020.602315

\section{Prevalence and Related Factors of Insomnia Among Chinese Medical Staff in the Middle and Late Stage of COVID-19}

\author{
Dianying Liu ${ }^{1 \dagger}$, Shaohua Liu ${ }^{1 \dagger}$, Lin Zhu ${ }^{1+}$, Dongbin $\mathrm{Li}^{2}$, Donghua Huang ${ }^{1}$, \\ Hongdong Deng ${ }^{1}$, Huiyun Guo ${ }^{1}$, Dan Huang ${ }^{1}$, Yuanping Liao ${ }^{1}$, Zhongzhen Mao ${ }^{1}$, \\ Qiumei Miao ${ }^{1}$, Wanglin Liu ${ }^{1}$, Meihong Xiu ${ }^{3 *}$ and Xiangyang Zhang ${ }^{4 *}$ \\ ${ }^{1}$ Department of Psychiatry, The Third People's Hospital of Ganzhou City, Ganzhou, China, ${ }^{2}$ School of Education Science, \\ Gannan Normal University, Ganzhou, China, ${ }^{3}$ Beijing HuiLongGuan Hospital, Peking University Huilong Guan Clinical Medical \\ School, Beijing, China, ${ }^{4}$ Chinese Academy of Sciences Key Laboratory of Mental Health, Institute of Psychology, Chinese \\ Academy of Sciences, Beijing, China
}

Background: The outbreak of novel coronavirus disease (COVID-19) has brought serious psychological pressure to people, especially medical health staff. At present, there are few studies on insomnia and related factors of medical health staff in the middle and late stage of the epidemic of COVID-19. Therefore, the purpose of this study was to investigate the prevalence of insomnia and its related risk factors among medical workers in China in the middle and later stage of COVID-19 epidemic, as well as the relationship between insomnia and psychological resilience.

Methods: From February 14 to March 29, 2020, a cross-sectional survey was conducted among 606 medical staff in China through Ranxing Technology's "SurveyStar" network platform. All subjects were assessed with the Insomnia Severity Index (ISI) and simplified Chinese version of Connor-Davidson Resilience scale (CD-RISC-10).

Results: In the middle and later stages of the COVID-19 outbreak, the incidence of insomnia among medical staff was 32.0\%. Compared with non-insomnia group, the insomnia group had younger age, lower education level, longer daily working hours and less psychological resilience. In addition, the prevalence of insomnia was higher in medical staff with a history of somatic diseases. The severity of insomnia of Chinese medical staff was associated with age, education level, daily working hours, psychological resilience and somatic diseases.

Conclusions: Our study shows that nearly $1 / 3$ of Chinese medical workers suffer from insomnia nearly a month after the COVID-19 outbreak. Compared with the general population, medical staff who are working with COVID are more prone to insomnia. Risk factors for insomnia include younger age, lower education level, longer working hours per day, and physical illness. The tenacious dimension of psychological resilience is a protective factor for insomnia.

Keywords: insomnia, COVID-19, prevalence, resilience, medical health staff 


\section{INTRODUCTION}

Insomnia is a major mental problem for medical staff, especially during disease outbreaks. Previous studies have shown that during the SARS epidemic, the percentage of insomnia among Chinese health care workers ranged from $37.1 \%$ (1) to $52.3 \%$ (2-4), which was 5-7 times (6-12.2\%) higher than the public population (2, 4, 5). In December 2019, the novel coronavirus disease (COVID-19) first appeared in Wuhan, Hubei, China (6), and then spread to other cities and even abroad (7). Because of its high infectivity and fatality rate, it brings tremendous psychological pressure to medical staff, which leads to an increase in the incidence of insomnia and seriously affects the mental health of medical staff $(8,9)$. In addition, insomnia is a major risk factor that increases depression, anxiety and suicide $(10,11)$.

A variety of common factors, such as age $(4,8,9,12,13)$, women $(4,9,12,13)$, low education level $(4,9,13)$, low income level (4), isolation environment (9), and marital status $(4,12,13)$ has been shown to be associated with personal insomnia. Moreover, another risk factor for insomnia among medical staff includes job position, such as nurse (9). Other studies have shown that long-term working hours and steadystate overload are important factors for increased insomnia among medical health workers $(1,8,9,14-16)$. Taken together, insomnia is caused by different risk factors, and insomnia is still a major health problem for medical workers. Most previous studies have focused on the relationship between insomnia and the sociodemographic variables, and only a few studies have investigated the relationship between insomnia and psychological resilience (17-21). Psychological resilience is the ability to adapt to stress and adversity. Li et al. (18) and Cheng et al. (21) found that people with low resilience directly lead to poor sleep quality. Similarly, Brand et al. (22) reported that compared with the high resilience group, the low resilience group has lower sleep efficiency, more awakenings, and lighter sleep. A recent study also found a significant positive correlation between the resilience and the quality of sleep in pregnant woman (17). However, most of those studies were conducted among nonmedical staff. Therefore, whether psychological resilience is a protective factor for sleep quality of medical staff is still unknown and deserves further study.

To our best knowledge, no study has reported the relationship between psychological resilience and insomnia of medical staff among Chinese Han population. Therefore, this study aimed to determine the prevalence and related factors of insomnia in Chinese medical staff through a cross-sectional design, including demographic data, daily working hours, somatic diseases, and psychological resilience.

\section{METHODS}

\section{Subjects and Settings}

A cross-sectional study was conducted from February 14 to March 29 2020. All questionnaires were distributed in the form of posters through the "SurveyStar" network platform (Ranxing Technology), which was forwarded through Wechat and other channels. The recruited medical staff logged in by scanning the
QR code and filled in the questionnaire. Finally, a total of 606 valid data were collected.

The research protocol was approved by the Ethics Committee of the Institute of Psychology, Chinese Academy of Sciences.

\section{Measurements}

A structured self-assessment questionnaire was conducted in this survey. Demographic data of all subjects were collected, including sex, age, height, weight, marital status, education level, occupation (doctors, nurses, or medical technicians), daily working hours, history of SARS epidemic in 2003, annual family income, history of somatic diseases, COVID-19 infection of relatives and friends, economic losses caused by COVID-19. The Chinese version of the Insomnia Severity Index (ISI) (23) was used to assess the severity of insomnia of all subjects. ISI is a selfreport scale composed of 7 items. Each item is evaluated on a five-point Likert scale (from $0=$ not at all to $4=$ extremely), and the usual recall interval is the "last 2 weeks." The total score ranges from 0 to 28 , and the higher the score, the greater the severity of insomnia. According to a previous study (24) a total score of 0-7 indicated no insomnia, 8-14 indicated subthreshold insomnia, 15-21 indicated moderate insomnia, and 22-28 indicated severe insomnia. In this survey, a total score of ISI $\geq 8$ indicated insomnia $(9,25)$.

Psychological resilience was measured by the simplified Chinese version of the 10- item Connor-Davidson Resilience Scale (CD-RISC-10). CD-RISC-10 is an important tool for assessing an individual's ability to cope with stress and rebound from difficult events or experiences (26), and has been widely used all over the world and in different populations (27). CDRISC-10 is a five-point Likert (from $0=$ never, $1=$ rarely, $2=$ sometimes, $3=$ often, and $4=$ always) and a self-rating scale. The usual recall interval is "the past month." The total score ranges from 0 to 40 , and a high total score indicates greater resilience (28). This scale includes two factors. One is strength, which mainly indicates the ability to deal with difficult problems, or the ability to achieve goals, and physical and mental resilience, which is composed of five items. The other is hardiness, which mainly refers to the ability to adapt to change in the face of setbacks or adversity, consisting of five items (29). The total score of these two factors ranges from 0 to 20 points.

\section{Statistical Analyses}

The demographic and mental health variables of insomnia and non-insomnia groups were compared using independent samples $t$-tests for continuous variables and chi-square for categorical variables. The prevalence of insomnia was described by percentage and analyzed by chi-square test. Binary logistic regression analysis was performed to assess which factors were significantly associated with insomnia. The correlation between insomnia and demographic and mental health variables was conducted by Pearson or Spearman correlation coefficients. Bonferroni correction was performed for each test to adjust for multiple tests. Then stepwise multiple regression analysis was applied to explore the significant predictive variables related to insomnia. IBM SPSS 22.0 was performed for all statistical 
analysis. All $p$-values were two tailed and the significance level was $<0.05$.

\section{RESULTS}

\section{Demographic and Mental Health Characteristics}

The demographic and mental health characteristics of all subjects are shown in Table 1. There were 114 males (18.8\%) and 492 females (81.2\%). The average age of all subjects was 35.77 years old, ranging from 20 to 65 years old. The average body mass index (BMI) was $22.76 \mathrm{~kg} / \mathrm{m}^{2}$, ranging from 12.36 to 47.27 $\mathrm{kg} / \mathrm{m}^{2} .152$ subjects (25.08\%) were single and $454(74.91 \%)$ were married. There were $80(13.2 \%)$ subjects with a college degree or below, $380(62.7 \%)$ with a bachelor degree, 92 (15.2\%) with a master degree, and $54(8.9 \%)$ with a doctoral degree. Among the jobs, there were 205 doctors (33.8\%), 334 nurses $(55.1 \%)$, and 67 medical technicians (11.1\%). Eighty-four subjects (46.9\%) worked $<8 \mathrm{~h}$ a day, 268 subjects (44.2\%) worked for $10 \mathrm{~h}$ or less, and 54 subjected $(8.9 \%)$ worked for more than $10 \mathrm{~h}$ a day. In addition, the annual household income of 106 subjects $(17.5 \%)$ was $30,000-80,000$ yuan, $402(66.3 \%)$ were $80,000-300,000$ yuan, and $98(16.2 \%)$ were $300,000-1,000,000$ yuan. In 2003, 262 subjects (43.2\%) experienced the SARS epidemic. 137 subjects (22.6\%) had a history of somatic disease. The average CD-RISC10 score of all subjects was 27.05. The average strength factor score was 13.66, and the average hardiness factor score was 13.39.

TABLE 1 | Demographic and mental health characteristics of medical staff with or without insomnia

\begin{tabular}{|c|c|c|c|c|c|}
\hline & Total & Non-insomnia group & Insomnia group & $t / \chi^{2}$ & $p$-Value \\
\hline & 606 & $(n=412,68.0 \%)$ & $(n=194,32.0 \%)$ & & \\
\hline Sex & & & & 0.11 & 0.739 \\
\hline Male, $n(\%)$ & $114(18.8)$ & 79/114 (69.30) & $35 / 114(30.70)$ & & \\
\hline Female, $n(\%)$ & $492(81.2)$ & $333 / 492(67.68)$ & 159/492 (32.32) & & \\
\hline Age, years, M (SD) & $35.77(8.13)$ & $38.45(8.08)$ & $34.33(8.07)$ & 3.01 & 0.003 \\
\hline BMI, M (SD) & $22.76(3.81)$ & $22.56(3.44)$ & $23.18(4.47)$ & -1.85 & 0.065 \\
\hline Marital status & & & & 5.19 & 0.023 \\
\hline Single, $n(\%)$ & $152(25.08)$ & $92 / 152(60.5)$ & 60/152 (39.5) & & \\
\hline Married, $n(\%)$ & $454(74.91)$ & $320 / 454(70.5)$ & $134 / 454(29.5)$ & & \\
\hline Education & & & & 9.59 & 0.022 \\
\hline College degree and below, $n$ (\%) & $80(13.2)$ & $45 / 80(56.2)$ & $35 / 80(43.8)$ & & \\
\hline Bachelor degree, $n(\%)$ & $380(62.7)$ & 256/380 (67.4) & $124 / 380(32.6)$ & & \\
\hline Master degree, $n(\%)$ & $92(15.2)$ & 69/92 (75.0) & 23/92 (25.0) & & \\
\hline Doctoral degree, $n(\%)$ & $54(8.9)$ & $42 / 54(77.8)$ & $12 / 54(22.2)$ & & \\
\hline Job position & & & & 12.56 & 0.002 \\
\hline Doctors & $205(33.8)$ & $153(74.6)$ & $52(25.4)$ & & \\
\hline Nurses & $334(55.1)$ & $207(62.0)$ & $127(38.0)$ & & \\
\hline Medical technicians & $67(11.1)$ & $52(77.6)$ & $15(22.4)$ & & \\
\hline Working hours/day (h) & & & & 12.09 & 0.002 \\
\hline$\leqq 8$ & $284(46.9)$ & $208(73.2)$ & $76(26.8)$ & & \\
\hline $8-10$ & $268(44.2)$ & $177(66.0)$ & $91(34.0)$ & & \\
\hline$>10$ & $54(8.9)$ & $27(50.0)$ & $27(50.0)$ & & \\
\hline History of SARS epidemic in 2003 & & & & 1.92 & 0.166 \\
\hline No & $344(56.8)$ & $226(65.7)$ & $118(34.3)$ & & \\
\hline Yes & $262(43.2)$ & $186(71.0)$ & $76(29.0)$ & & \\
\hline Annual household incomes (Yuan) & & & & 7.03 & 0.03 \\
\hline $30,000-80,000$ & $106(17.5)$ & $61(57.5)$ & $45(42.5)$ & & \\
\hline $80,000-300,000$ & $402(66.3)$ & $279(69.4)$ & $123(30.6)$ & & \\
\hline $300,000-\geq 1,000,000$ & $98(16.2)$ & $72(73.5)$ & $26(26.5)$ & & \\
\hline History somatic disease & & & & 8.67 & 0.003 \\
\hline No & $469(77.4)$ & $333(71.0)$ & $136(29.0)$ & & \\
\hline yes & $137(22.6)$ & $79(57.7)$ & $58(42.3)$ & & \\
\hline \multicolumn{6}{|l|}{ CD-RISC-10 } \\
\hline Total score, M (SD) & $27.05(8.71)$ & $28.67(8.65)$ & $23.60(7.80)$ & 6.94 & $<0.001$ \\
\hline Strength factor, M (SD) & $13.66(4.51)$ & $14.45(4.47)$ & $11.97(4.13)$ & 6.51 & $<0.001$ \\
\hline Hardiness factor, M (SD) & $13.39(4.35)$ & $14.22(4.33)$ & $11.63(3.84)$ & 7.12 & $<0.001$ \\
\hline
\end{tabular}




\section{Prevalence of Insomnia and Comparison of Demographic and Mental Health Variables Between Insomnia and Non-insomnia Participants}

As shown in Table 1, the prevalence of insomnia among medical staff was 32.0\% (194/606). The average age of the insomnia group was significantly younger than that of the non-insomnia group $(p=0.003)$. The prevalence of insomnia in single subjects was significantly higher than that in married subjects $(p=0.023)$. The prevalence of insomnia in college degree and below was significantly higher $43.8 \%$ (35/80) than that bachelor degree $32.6 \%$ (124/380), master degree 25.0\% (23/92) and doctoral degree 22.2\% (12/54) $(p=0.022$, Bonferroni corrected $p<$ $0.05)$. The prevalence of insomnia among nurses was significantly higher than that in doctors and medical technicians $(p=0.002$, Bonferroni corrected $p<0.01$ ). The incidence of insomnia in the group working more than $10 \mathrm{~h}$ a day was significantly higher than that in the $8-10 \mathrm{~h}$ group and the $8 \mathrm{~h}$ group $(p=0.002$, Bonferroni corrected $p<0.01$ ). The rate of insomnia in medical staff with somatic disease was significantly higher than that in non-somatic disease group ( $p=0.003)$.

The total score, strength factor and hardiness factor scores of CD-RISC-10 in insomnia group were significantly lower than those in non-insomnia group (all $p<0.001$ ). There was no significant difference in BMI, history of SARS epidemic in 2003 (all $p>0.05$ ). The lowest annual household incomes group had significantly highest rate of insomnia $(p=0.03)$ (Table 1). In addition, after controlling for gender as a covariate, these differences remained significant (all $p<0.05$ ).

\section{Correlation of Insomnia and Demographic and Mental Health Measures}

The average ISI total score in all medical staff was $6.27 \pm 6.13$. Pearson correlation analysis showed that ISI was correlated with age $(r=-0.134, p<0.001)$, marital status $(r=-0.086, p<0.05)$, education level $(r=-0.143, p<0.001)$, daily working hours $(r$ $=0.1, p<0.01)$, physical illness $(r=0.095, p<0.05)$, strength factor $(r=-0.304, p<0.001)$, and hardiness factor $(r=-0.327$, $p<0.001)$. Further, except for marital status, all these associates remained significant $(p<0.05)$ after the Bonferroni correction. Table 2 shows the association between ISI and demographic data or mental health variables.

\section{Factors Associated With Insomnia}

Multiple stepwise regression was performed to identify demographic and mental health variables that were associated with ISI. There were five variables that statistically predicted ISI $\left[F_{(5,600)}=20.05, p<0.001, r^{2}=0.136\right]$, including hardiness factor, daily working hours, education level, physical illness, and age. The coefficients of these variables are shown in Table 3.

\section{DISCUSSION}

To our best knowledge, this is the first study to investigate the percentage of insomnia and its related risk factors among medical staff under the long-term influence of COVID-19 pandemic in China, as well as the relationship between insomnia and psychological resilience. The main findings of this survey included: (1) the percentage of insomnia in medical staff was 32.0\%; (2) medical staff working with COVID were more prone to insomnia than the general population; (3) the risk factors of insomnia in medical staff were younger age, lower education level, longer working hours per day, and physical illness; (4) hardiness factor of psychological resilience was the protective factor for insomnia of medical staff.

Our cross-sectional study indicated that during the COVID19 epidemic in China, the percentage of medical staff who suffered from insomnia was $32.0 \%$, which was lower than the previous studies of $34.0 \%$ (30), 36.1\% (9), 38.4\% (31), but was relatively higher than the $30.5 \%$ prevalence of non-medical personnel under the COVID-19 epidemic (31). The difference in the incidence of insomnia was most likely to be related to the following reasons. First, contrary to previous studies, the duration of our investigation was longer, from February 14 to March 29, 2020. At the beginning of the COVID-19 outbreak, medical staff lacked awareness of the disease and lacked protective equipment, which increased their anxiety, fear, and insomnia. With the spread of the COVID-19, medical staff has had a better understanding of the disease, treatment for the disease has been improved, and anxiety, fear and insomnia have been alleviated. Second, more medical staff have been sent to Wuhan, reducing the pressure on medical staff. Third, the government and various units have taken a series of timely and effective psychosocial interventions and support measures (6). Taken together, these studies have shown that with the progression of the COVID-19 epidemic, the prevalence of insomnia gradually decreases, but is still higher in medical staff than that of the Chinese public during the COVID-19 epidemic.

Furthermore, our study found that the ISI total score was negatively correlated with age in Chinese medical staff, which is consistent with previous studies showing that medical staff in the insomnia group were between 18 and 25 years old (9). As pointed out by Huang and Zhao (8), during COVID-19 outbreak, younger participants are more likely to suffer from anxiety and depressive than older people. The possible reason is that young medical have relatively lack of clinical experience in the face of inadequate working environment, including long waiting lists of patients, heavy workload, insufficient resources, and daily working overload, which can easily lead to anxiety, depression, insomnia and other problems. But gender was not related to the insomnia during COVID-19 outbreak, which was consistent with previous studies $(8,9)$.

In addition, we found that marital status was associated with insomnia, which is in line with a previous study (9) reporting that the insomnia rate of single medical staff was higher than that of married subjects (38.1 vs. $34.43 \%$ ). However, other studies showed that married participants have a higher rate of insomnia than single participants $(12,13,32)$. The possible reason for these inconsistent results is that the participants come from different places. The subjects recruited in this study and Zhang et al. (9) study were hospital staff from all over the country, including front-line medical staff. But the subjects of Li et al. (32) all came from Ningbo city. In another study, the subjects were from the 
TABLE 2 | Association between ISI and demographic data and mental health variables.

\begin{tabular}{|c|c|c|c|c|c|c|c|c|c|c|c|c|}
\hline Variables & ISI & Sex & Age & Marital & Education & $\begin{array}{c}\text { Job } \\
\text { position }\end{array}$ & $\begin{array}{l}\text { Working } \\
\text { duration }\end{array}$ & $\begin{array}{c}\text { Annual } \\
\text { incomes }\end{array}$ & $\begin{array}{l}\text { Somatic } \\
\text { disease }\end{array}$ & Resilience & Strength & Hardiness \\
\hline ISI & 1 & & & & & & & & & & & \\
\hline Sex & 0.052 & 1 & & & & & & & & & & \\
\hline Age & $-0.134^{\star \star \star}$ & $-0.172^{\star \star \star}$ & 1 & & & & & & & & & \\
\hline Marital & $-0.086^{\star}$ & -0.074 & $0.480^{\star \star \star}$ & 1 & & & & & & & & \\
\hline Education degree & $-0.143^{\star \star \star}$ & $-0.193^{\star \star}$ & $0.275^{\star \star \star}$ & $0.158^{\star \star \star}$ & 1 & & & & & & & \\
\hline Job position & 0.069 & $0.322^{\star \star}$ & $-0.220^{\star \star \star}$ & $-0.119^{\star \star}$ & $-0.381^{\star \star \star}$ & 1 & & & & & & \\
\hline Working duration & $0.100^{\star \star}$ & $-0.081^{*}$ & $0.115^{\star \star}$ & 0.031 & $0.131^{\star \star}$ & $-0.185^{\star \star \star}$ & 1 & & & & & \\
\hline Annual incomes & -0.070 & -0.011 & $0.295^{\star \star \star}$ & $0.249^{\star \star \star}$ & $0.376^{\star \star \star}$ & $-0.117^{\star \star \star}$ & $0.115^{\star *}$ & 1 & & & & \\
\hline Somatic disease & $0.095^{\star}$ & -0.002 & $0.217^{\star \star \star}$ & $0.103^{\star \star}$ & 0.035 & -0.049 & $0.092^{\star}$ & $0.094^{*}$ & 1 & & & \\
\hline Resilience & $-0.321^{\star \star \star}$ & -0.064 & $0.218^{\star \star \star}$ & 0.045 & $0.176^{\star \star \star}$ & -0.053 & 0.011 & $0.176^{\star \star \star}$ & 0.022 & 1 & & \\
\hline Strength & $-0.304^{\star \star \star}$ & -0.050 & $0.210^{\star \star \star}$ & 0.037 & $0.167^{\star \star \star}$ & -0.049 & 0.006 & $0.178^{\star \star \star}$ & 0.020 & $0.983^{\star \star \star}$ & 1 & \\
\hline Hardiness & $-0.327^{\star \star \star}$ & -0.076 & $0.217^{\star \star \star}$ & 0.051 & $0.179^{\star \star \star}$ & -0.055 & 0.16 & $0.168^{\star \star \star}$ & 0.024 & $0.982^{\star \star \star}$ & $0.932^{\star \star \star}$ & 1 \\
\hline
\end{tabular}

${ }^{\star}$ Indicates that there was a significant corelation. ${ }^{*} P<0.05 ;{ }^{* \star} p<0.01 ;{ }^{\star \star *} p<0.001$.

TABLE 3 | Predictors generated by multivariate logistic regression with ISI total score as dependent variables.

\begin{tabular}{|c|c|c|c|c|c|c|c|}
\hline & \multicolumn{2}{|c|}{ Coefficients } & \multirow{2}{*}{$\begin{array}{c}\begin{array}{l}\text { Standardized } \\
\text { coefficients }\end{array} \\
\text { Beta }\end{array}$} & \multirow[t]{2}{*}{$T$} & \multirow[t]{2}{*}{$p$-Value } & \multicolumn{2}{|c|}{$95.0 \%$ confidence interval for $\mathrm{B}$} \\
\hline & $B$ & Std. Error & & & & $\begin{array}{l}\text { Lower } \\
\text { bound }\end{array}$ & Upper bound \\
\hline (Constant) & 13.479 & 1.290 & & 10.450 & 0.000 & 10.946 & 16.012 \\
\hline Hardiness factor & -0.420 & 0.055 & -0.298 & -7.636 & 0.000 & -0.529 & -0.312 \\
\hline Working hours/day & 1.097 & 0.365 & 0.115 & 3.004 & 0.003 & 0.380 & 1.815 \\
\hline Education & -0.679 & 0.315 & -0.086 & -2.154 & 0.032 & -1.297 & -0.060 \\
\hline Somatic disease & 1.652 & 0.569 & 0.113 & 2.904 & 0.004 & 0.535 & 2.769 \\
\hline Age & -0.063 & 0.031 & -0.083 & -2.022 & 0.044 & -0.123 & -0.002 \\
\hline
\end{tabular}

general population (13). Therefore, it is necessary to further investigate the differences in the incidence of insomnia among medical staff with different marital status. In addition, we found that medical staff with lower education level were at a higher risk of insomnia, which was consistent with several previous studies $(9,13)$ showing that low education level was associated with a high risk of insomnia in the general population and medical staff in China. The main reason is that it is more difficult for medical staff with low education level to grasp the information related to disease outbreaks, and their working ability is relatively poor. During the COVID-19 outbreak, they had a stronger fear of the disease, which may affect their sleep quality (9). It is worth noting that our study further indicated that medical staff who had longer working hours per day reported more serious symptoms of insomnia. This was in line with previous studies showing that medical staff who worked longer than usual reported more severe insomnia $(30,33)$. A potential explanation is that during the COVID-19 epidemic, both frontline and non-frontline medical staff spent a lot of time participating in the antiepidemic work, irregular shifts, excessive workload and longer working hours made them more stressed than usual $(16,32)$. These stresses constituted a source of unbalanced load, leading to burnout syndrome (BS) (34) and may significantly impair sleep $(15,31)$. As expected, insomnia was significantly associated with somatic diseases in medical staff, and with the increase in comorbid somatic diseases in the entire sample, the prevalence of insomnia became higher. Consistent with other studies, patients with chronic physical diseases have an increased risk of insomnia $(31,35)$. Whether medical staff or non-medical staff, organic diseases are independent risk factors for insomnia (9). One possible explanation is that physical complaints, such as headache and cardiovascular disease are more likely to enhance autonomic hyperarousal. Second, during the COVID-19 epidemic, medical staff with physical diseases may worry about infection, resulting in more serious symptoms of anxiety and insomnia. Moreover, negative thoughts about threatening symptoms may lead to persistent insomnia (36).

Another important finding of this study was that all the dimensions and total score of psychological resilience were significantly negatively correlated with the ISI total score. Our study also showed that non-insomnia medical staff had better psychological resilience and stronger strength and hardiness. This was in accordance with other studies showing that participants with low resilience directly lead to poor sleep quality 
$(17,20)$, such as lower sleep efficiency, more awakening times, and lighter sleep than those with higher resilience (22).

However, in this study, multiple stepwise regression found that only the hardiness subscale of the psychological resilience was the significant protective factor for insomnia in medical workers. Hardiness reflects a person's ability to rebound from adversity, emotional control, decision-making, and problemsolving. Previous study reported that hardiness was a protective factor for negative health outcomes among the five factors of resilience (hardiness, optimism, persistence, support, and spirituality) (37). These findings indicate that psychological resilience is a protective factor for insomnia in medical staff.

Our research had several limitations. First, this survey adopted a cross-sectional design, based on the WeChat program and selfadministered questionnaire. Second, the duration of the survey is comparatively short, which cannot effectively verify whether there is dynamic balance overload as COVID-19 progresses. Third, only ISI was used to assess the severity of insomnia. It is possible that many other sleep problems had not been assessed. Fourth, we did not compare the difference in insomnia between frontline and non-frontline medical workers. It is not obvious whether the data on insomnia in medical staff working with COVID is different from the insomnia in medical workers dealing with routine diseases. Fifth, the pre-epidemic status was not collected, which may lead to biased results. In addition, the most important limitation is the source of recruitment. This sample could be not representative because the older medical staff could not use social networks. This is evident because of low mean age of participants (35 years).

In conclusion, this study revealed that during the COVID19 outbreak, the prevalence of insomnia among medical staff was higher than that of the Chinese public. The related risk factors included younger age, lower education level, physical disease, and longer working hours per day. In addition, the hardiness of psychological resilience was a protective factor for insomnia of medical staff. Therefore, when carrying on

\section{REFERENCES}

1. Su TP, Lien TC, Yang CY, Su YL, Wang JH, Tsai SL, et al. Prevalence of psychiatric morbidity and psychological adaptation of the nurses in a structured SARS caring unit during outbreak: a prospective and periodic assessment study in Taiwan. J Psychiatr Res. (2007) 41:11930. doi: 10.1016/j.jpsychires.2005.12.006

2. Wu P, Fang Y, Guan Z, Fan B, Kong J, Yao Z, et al. The psychological impact of the SARS epidemic on hospital employees in China exposure, risk perception, and altruistic acceptance of risk. Can J Psychiatry. (2009) 54:302-11. doi: 10.1177/070674370905400504

3. Chong MY, Wang WC, Hsieh WC, Lee CY, Chiu NM, Yeh WC, et al. Psychological impact of severe acute respiratory syndrome on health workers in a tertiary hospital. Br J Psychiatry. (2004) 185:12733. doi: 10.1192/bjp.185.2.127

4. Benbir G, Demir AU, Aksu M, Ardic S, Firat H, Itil O, et al. Prevalence of insomnia and its clinical correlates in a general population in Turkey. Psychiatry Clin Neurosci. (2015) 69:543-52. doi: 10.1111/pcn.12252

5. Xiang YT, Jin Y, Wang Y, Zhang Q, Zhang L, Cheung T. Tribute to health workers in China: a group of respectable population during the outbreak of the COVID-19. Int J Biol Sci. (2020) 16:1739-40. doi: 10.7150/ ijbs. 45135 the psychological intervention to the medical staff, we need to consider different social and psychological factors. The most important intervention is to improve the psychological adaptability of medical staff.

\section{DATA AVAILABILITY STATEMENT}

The original contributions presented in the study are included in the article/supplementary material, further inquiries can be directed to the corresponding author/s.

\section{ETHICS STATEMENT}

The studies involving human participants were reviewed and approved by the Ethics Committee of the Institute of Psychology, Chinese Academy of Sciences. The patients/participants provided their written informed consent to participate in this study.

\section{AUTHOR CONTRIBUTIONS}

XZ: conceptualization, methodology, writing-reviewing, editing, and supervision. DLiu: formal analysis, writingoriginal draft, and funding acquisition. SL, LZ, DLi, DoH, $\mathrm{HD}, \mathrm{HG}, \mathrm{DaH}, \mathrm{YL}$, and ZM: investigation, resources, and data curation. WL, QM, and MX: conceptualization, writing-review, and editing. All authors contributed to the article and approved the submitted version.

\section{FUNDING}

This study were supported by the Spark Program of Health Commission of Jiangxi Province of China (20208054), the Key Research and Development Project of Science and Technology in Ganzhou (201926), and the Planning Project of Health Committee of Ganzhou (201911). All funds have no role in research design, data analysis, paper submission, and publication.
6. Zhou Y, Zhou Y, Song Y, Ren L, Ng CH, Xiang YT, et al. Tackling the mental health burden of frontline healthcare staff in the COVID-19 pandemic: China's experiences. Psychol Med. (2020) 13:1-5. doi: 10.1017/S0033291720001622

7. Wang WJ, Hou CL, Jiang YP, Han FZ, Wang XY, Wang SB, et al. Prevalence and associated risk factors of insomnia among pregnant women in China. Compr Psychiatry. (2020) 98:152168. doi: 10.1016/j.comppsych.2020.152168

8. Huang Y, Zhao N. Generalized anxiety disorder, depressive symptoms and sleep quality during COVID-19 outbreak in China: a web-based cross-sectional survey. Psychiatry Res. (2020) 288:112954. doi: 10.1016/j.psychres.2020.112954

9. Zhang C, Yang L, Liu S, Ma S, Wang Y, Cai Z, et al. Survey of insomnia and related social psychological factors among medical staff involved in the 2019 novel coronavirus disease outbreak. Front Psychiatry. (2020) 11:306. doi: 10.3389/fpsyt.2020.00306

10. Baglioni C, Battagliese G, Feige B, Spiegelhalder K, Nissen C, Voderholzer $\mathrm{U}$, et al. Insomnia as a predictor of depression: a meta-analytic evaluation of longitudinal epidemiological studies. J Affect Disord. (2011) 135:109. doi: 10.1016/j.jad.2011.01.011

11. Palagini L, Cipollone G, Masci I, Novi M, Caruso D, Kalmbach DA, et al. Stress-related sleep reactivity is associated with insomnia, psychopathology and suicidality in pregnant women: preliminary results. Sleep Med. (2019) 56:145-50. doi: 10.1016/j.sleep.2019.01.009 
12. Leger D, Guilleminault C, Dreyfus JP, Delahaye C, Paillard M. Prevalence of insomnia in a survey of 12,778 adults in France. J Sleep Res. (2000) 9:35-42. doi: 10.1046/j.1365-2869.2000.00178.x

13. Xiang YT, Ma X, Cai ZJ, Li SR, Xiang YQ, Guo HL, et al. The prevalence of insomnia, its sociodemographic and clinical correlates, and treatment in rural and urban regions of Beijing, China: a general population-based survey. Sleep. (2008) 31:1655-62. doi: 10.1093/sleep/31.12.1655

14. Lung FW, Lu YC, Chang YY, Shu BC. Mental symptoms in different health professionals during the SARS attack: a follow-up study. Psychiatr Q. (2009) 80:107-16. doi: 10.1007/s11126-009-9095-5

15. Fava GA, McEwen BS, Guidi J, Gostoli S, Offidani E, Sonino N. Clinical characterization of allostatic overload. Psychoneuroendocrinology. (2019) 108:94-101. doi: 10.1016/j.psyneuen.2019.05.028

16. Kang L, Ma S, Chen M, Yang J, Wang Y, Li R, et al. Impact on mental health and perceptions of psychological care among medical and nursing staff in Wuhan during the 2019 novel coronavirus disease outbreak: a cross-sectional study. Brain Behav Immun. (2020) 87:11-7. doi: 10.1016/j.bbi.2020.03.028

17. Li G, Kong L, Zhou H, Kang X, Fang Y, Li P. Relationship between prenatal maternal stress and sleep quality in Chinese pregnant women: the mediation effect of resilience. Sleep Med. (2016) 25:8-12. doi: 10.1016/j.sleep.2016.02.015

18. Seelig AD, Jacobson IG, Donoho CJ, Trone DW, Crum-Cianflone NF, Balkin TJ. Sleep and health resilience metrics in a large military cohort. Sleep. (2016) 39:1111-20. doi: 10.5665/sleep.5766

19. Garbarino S, Magnavita N. Sleep problems are a strong predictor of stressrelated metabolic changes in police officers. a prospective study. PLOS ONE. (2019) 14:e0224259. doi: 10.1371/journal.pone.0224259

20. Cheng MY, Wang MJ, Chang MY, Zhang RX, Gu CF, Zhao YH. Relationship between resilience and insomnia among the middle-aged and elderly: mediating role of maladaptive emotion regulation strategies. Psychol Health Med. (2020) 10:1-12. doi: 10.1080/13548506.2020.1734637

21. Yang $X$, You L, Jin D, Zou X, Yang H, Liu T. A communitybased cross-sectional study of sleep quality among internal migrant workers in the service industry. Compr Psychiatry. (2020) 97:152154. doi: 10.1016/j.comppsych.2019.152154

22. Brand S, Gerber M, Kalak N, Kirov R, Lemola S, Clough PJ, et al. Adolescents with greater mental toughness show higher sleep efficiency, more deep sleep and fewer awakenings after sleep onset. J Adolesc Health. (2014) 54:10913. doi: 10.1016/j.jadohealth.2013.07.017

23. Morin CM. Insomnia: Psychological Assessment and Management. New York, NY: Guilford Press (1993).

24. Morin CM, Belleville G, Bélanger L, Ivers H. The Insomnia Severity Index: psychometric indicators to detect insomnia cases and evaluate treatment response. Sleep. (2011) 34:601-8. doi: 10.1093/sleep/34.5.601

25. Wong SY, Zhang DX, Li CC, Yip BH, Chan DC, Ling YM, et al. Comparing the effects of mindfulness-based cognitive therapy and sleep psycho-education with exercise on chronic insomnia: a randomised controlled trial. Psychother Psychosom. (2017) 86:241-53. doi: 10.1159/000470847

26. Cohen S, Kamarck T, Mermelstein R. A global measure of perceived stress. $J$ Health Soc Behav. (1983) 24:385-96.

27. Cheng C, Dong D, He J, Zhong X, Yao S. Psychometric properties of the 10-item Connor-Davidson Resilience Scale (CD-RISC-10) in Chinese undergraduates and depressive patients. J Affect Disord. (2020) 261:21120. doi: 10.1016/j.jad.2019.10.018

28. Campbell-Sills L, Stein MB. Psychometric analysis and refinement of the Connor-davidson Resilience Scale (CD-RISC): validation of a 10-item measure of resilience. J Trauma Stress. (2007) 20:1019-28. doi: 10.1002/jts.20271

29. Zhang Danmei XM, Yanzhang L. The reliability and validity of 10-item Connor-Davidson resilience scale in the community-dwelling older adults. Aging Ment Health. (2018) 27:942-6. doi: 10.1080/13607863.2019.1683812

30. Lai J, Ma S, Wang Y, Cai Z, Hu J, Wei N, et al. Factors associated with mental health outcomes among health care workers exposed to coronavirus disease 2019. JAMA Netw Open. (2020) 3:e203976. doi: 10.1001/jamanetworkopen.2020.3976

31. Zhang WR, Wang K, Yin L, Zhao WF, Xue Q, Peng M, et al. Mental health and psychosocial problems of medical health workers during the COVID19 epidemic in China. Psychother Psychosom. (2020) 4:1-9. doi: 10.1159/0005 07639

32. Li X, Yu H, Bian G, Hu Z, Liu X, Zhou Q, et al. Prevalence, risk factors, and clinical correlates of insomnia in volunteer and at home medical staff during the COVID-19. Brain Behav Immun. (2020) 87:1401. doi: 10.1016/j.bbi.2020.05.008

33. Shih FJ, Gau ML, Kao CC, Yang CY, Lin YS, Liao YC, et al. Dying and caring on the edge: Taiwan's surviving nurses' reflections on taking care of patients with severe acute respiratory syndrome. Appl Nurs Res. (2007) 20:171-80. doi: 10.1016/j.apnr.2006.08.007

34. Christina Maslach WBS, Leiter MP. Job burnout. Annu Rev Psychol. (2001) 52:397-422. doi: 10.1146/annurev.psych.52.1.397

35. Crönlein $T$, Langguth $B$, Pregler $M$, Kreuzer PM, Wetter TC, Schecklmann M. Insomnia in patients with chronic tinnitus: cognitive and emotional distress as moderator variables. J Psychosom Res. (2016) 83:65-8. doi: 10.1016/j.jpsychores.2016.03.001

36. Marks E, Hallsworth C, McKenna L. Cognitive behavioural therapy for insomnia (CBTi) as a treatment for tinnitus-related insomnia: protocol for a randomised controlled trial. Trials. (2019) 20:667. doi: 10.1186/s13063-019-3778-5

37. Shin YC, Kim SM, Kim H, Min KJ, Yoo SK, Kim EJ, et al. Resilience as a protective factor for depressive mood and anxiety among Korean employees. J Korean Med Sci. (2019) 34:e188. doi: 10.3346/jkms.2019. 34.e188

Conflict of Interest: The authors declare that the research was conducted in the absence of any commercial or financial relationships that could be construed as a potential conflict of interest.

Copyright (c) $2020 \mathrm{Liu}, \mathrm{Liu}, \mathrm{Zhu}, \mathrm{Li}$, Huang, Deng, Guo, Huang, Liao, Mao, Miao, Liu, Xiu and Zhang. This is an open-access article distributed under the terms of the Creative Commons Attribution License (CC BY). The use, distribution or reproduction in other forums is permitted, provided the original author(s) and the copyright owner(s) are credited and that the original publication in this journal is cited, in accordance with accepted academic practice. No use, distribution or reproduction is permitted which does not comply with these terms. 